\title{
CHARACTERIZATIONS OF LOCALLY FINITE ACTIONS OF GROUPS ON SETS
}

\author{
EDUARDO SCARPARO
}

\begin{abstract}
We show that an action of a group on a set $X$ is locally finite if and only if $X$ is not equidecomposable with a proper subset of itself. As a consequence, a group is locally finite if and only if its uniform Roe algebra is finite.
\end{abstract}

\section{INTRODUCTION}

Given a group acting on a set $X$, a property that has been well-studied is the existence of an invariant mean on $X$, that is, amenability of the action (see [1] for historical remarks). By Tarski's Theorem ([6, Corollary 9.2]), this is equivalent to $X$ not being equidecomposable with two disjoint subsets of itself.

In this note, we address the following question: given an action of a group $G$ on a set $X$, when is $X$ not equidecomposable with a proper subset of itself? We show that this holds if and only if the action is locally finite (Definition 2.2), if and only if $\ell^{\infty}(X) \rtimes_{r} G$ is a finite $\mathrm{C}^{*}$-algebra (Theorem 2.3). It follows from this that a group is locally finite if and only if its uniform Roe algebra $\left(\ell^{\infty}(G) \rtimes_{\mathrm{r}} G\right)$ is finite (Proposition 2.5). In [3], it was shown that $\ell^{\infty}(G) \rtimes_{\mathrm{r}} G$ is finite if $G$ is locally finite and asked if the converse holds.

It was already known that amenability of a group $G$ is equivalent to $\ell^{\infty}(G) \rtimes_{\mathrm{r}}$ $G$ not being properly infinite, and supramenability is equivalent to $\ell^{\infty}(G) \rtimes_{\mathrm{r}} G$ not containing any properly infinite projections ([3, Proposition 5.3]). Therefore, Proposition 2.5 completes the dictionary between equidecomposition properties of groups and the type of projections in the uniform Roe algebra.

\section{Characterizations of LOCAlly Finite aCtions of Groups on SETS}

We start by recalling some definitions:

Definition 2.1. Let be $G$ be a group acting on a set $X$. Two subsets $A$ and $B$ of $X$ are said to be equidecomposable if there are finite partitions $\left\{A_{i}\right\}_{i=i}^{n}$ and $\left\{B_{i}\right\}_{i=i}^{n}$ of $A$ and $B$, respectively, and elements $s_{1}, \ldots, s_{n} \in G$ such that $B_{i}=s_{i} A_{i}$ for $1 \leq i \leq n$. When we say that two subsets of $G$ are equidecomposable, it is with respect to the left action of $G$ on itself.

The next definition has already been introduced in [5] for actions on semilattices.

Definition 2.2. An action of a group $G$ on a set $X$ is said to be locally finite if, for every finitely generated subgroup $H$ of $G$ and every $x \in X$, the $H$-orbit of $x$ is finite.

This work was supported by CNPq, National Council for Scientific and Technological Development - Brazil. 
The left action of a group on itself is locally finite if and only if the group is locally finite.

The following result shows that the notion of locally finite action is a natural strengthening of the notion of amenable action on a set.

Theorem 2.3. Let $G$ be a group acting on a set $X$. The following conditions are equivalent:

(1) The action is locally finite;

(2) $\ell^{\infty}(X) \rtimes_{r} G$ is finite;

(3) $X$ is not equidecomposable with a proper subset of itself;

(4) No subset of $X$ is equidecomposable with a proper subset of itself.

Proof. (1) $\Rightarrow(2)$. Since the inductive limit of finite unital $\mathrm{C}^{*}$-algebras with unital conneting maps is finite, it suffices to show that $\ell^{\infty}(X) \rtimes_{\mathrm{r}} H$ is finite for every finitely generated subgroup $H$ of $G$. Let $H$ be such a subgroup and $X=\sqcup_{i \in I} X_{i}$ be the partition of $X$ into its $H$-orbits.

For every $i \in I$, the restriction map $\ell^{\infty}(X) \rightarrow \ell^{\infty}\left(X_{i}\right)$ is $H$-equivariant. Therefore, there is a homomorphism $\psi: \ell^{\infty}(X) \rtimes_{\mathrm{r}} H \rightarrow \prod\left(\ell^{\infty}\left(X_{i}\right) \rtimes_{\mathrm{r}} H\right)$. We claim that $\psi$ is injective.

Let $\varphi: \ell^{\infty}(X) \rtimes_{\mathrm{r}} H \rightarrow \ell^{\infty}(X)$ and, for every $i \in I, \varphi_{i}: \ell^{\infty}\left(X_{i}\right) \rtimes_{\mathrm{r}} H \rightarrow \ell^{\infty}\left(X_{i}\right)$ be the canonical conditional expectations. Also let $\varphi_{I}: \prod\left(\ell^{\infty}\left(X_{i}\right) \rtimes_{\mathrm{r}} H\right) \rightarrow \prod \ell^{\infty}\left(X_{i}\right)$ be the product of the maps $\varphi_{i}$, and $T: \ell^{\infty}(X) \rightarrow \prod \ell^{\infty}\left(X_{i}\right)$ be the isomorphism which arises from the product of the restriction maps. The following diagram commutes:

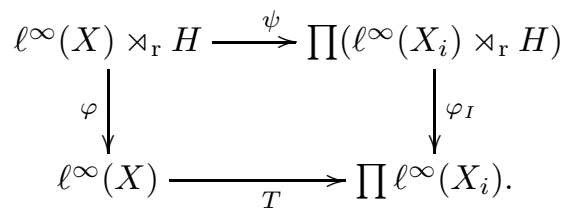

Since $\varphi$ is faithful, we conclude that $\psi$ is injective. Since the product of finite unital $\mathrm{C}^{*}$-algebras is finite, it suffices to show that $\ell^{\infty}\left(X_{i}\right) \rtimes_{\mathrm{r}} H$ is finite for every $i \in I$ in order to conclude that $\ell^{\infty}(X) \rtimes_{\mathrm{r}} H$ is finite.

Given $i \in I$, let $\tau_{i}$ be the tracial state on $\ell^{\infty}\left(X_{i}\right)$ which arises from the uniform probability measure on the finite set $X_{i}$. Since $\tau_{i}$ is $H$-invariant and faithful, it follows that the map $\tau_{i} \circ \varphi_{i}: \ell^{\infty}\left(X_{i}\right) \rtimes_{\mathrm{r}} H \rightarrow \mathbb{C}$ is a faithful tracial state. Therefore, $\ell^{\infty}\left(X_{i}\right) \rtimes_{\mathrm{r}} H$ is finite.

$(2) \Rightarrow(3)$. This follows from the fact that, if $A$ and $B$ are equidecomposable subsets of $X$, then the projections $1_{A}$ and $1_{B}$ are equivalent in $\ell^{\infty}(X) \rtimes_{\mathrm{r}} G$.

$(3) \Rightarrow(4)$. If $A \subset X$ is equidecomposable with $B \subsetneq A$, then $X=A \sqcup A^{c}$ is equidecomposable with $B \sqcup A^{c} \subsetneq X$.

$(4) \Rightarrow(1)$. Suppose that there is $H<G$ generated by a finite and symmetric set $S$ and $x \in X$ such that $H x$ is infinite. Then there exists a sequence $\left(s_{n}\right)_{n \in \mathbb{N}} \subset S$ such that

$$
\forall n, m \in \mathbb{N}: n \neq m \Rightarrow s_{n} \cdots s_{1} x \neq s_{m} \cdots s_{1} x .
$$

The sequence $\left(s_{n} \cdots s_{1} x\right)_{n \in \mathbb{N}}$ can be seen as an infinite simple path in the graph whose vertex set is $H x$ and whose edges come from $S$.

We claim that $\gamma:=\left\{s_{n} \cdots s_{1} x: n \in \mathbb{N}\right\}$ is equidecomposable with $\gamma \backslash\left\{s_{1} x\right\}$. 
Given $s \in S$, let $A_{s}:=\left\{s_{n} \cdots s_{1} x: s_{n+1}=s\right\}$. It is easy to check that $\left\{A_{s}\right\}_{s \in S}$ partitions $\gamma$ and $\left\{s A_{s}\right\}_{s \in S}$ partitions $\gamma \backslash\left\{s_{1} x\right\}$. Hence, $\gamma$ is equidecomposable with its proper subset $\gamma \backslash\left\{s_{1} x\right\}$.

We now proceed to give a characterization of locally finite groups which can be seen as an analogy to parts of [3, Theorem 1.1].

The next definition is from [4.

Definition 2.4. Let $H$ and $G$ be groups. A map $f: H \rightarrow G$ is said to be a uniform embedding if, for every finite set $S \subset H$, there is a finite set $T \subset G$ such that:

$$
\forall x, y \in H: x y^{-1} \in S \Longrightarrow f(x) f(y)^{-1} \in T
$$

and, for every finite set $T \subset G$, there is $S \subset H$ finite such that

$$
\forall x, y \in H: f(x) f(y)^{-1} \in T \Longrightarrow x y^{-1} \in S .
$$

The implication $(1) \Rightarrow(2)$ in the next result had already been observed in 3 , Remark 5.4], and (5) $\Rightarrow(1)$ is an immediate consequence of [8, Lemma 1].

Proposition 2.5. Let $G$ be a group. The following conditions are equivalent:

(1) $G$ is locally finite;

(2) The uniform Roe algebra $\ell^{\infty}(G) \rtimes_{\mathrm{r}} G$ is finite;

(3) $G$ is not equidecomposable with a proper subset of itself;

(4) No subset $A \subset G$ is equidecomposable with a proper subset of itself;

(5) There is no injective uniform embedding from $\mathbb{Z}$ into $G$.

Proof. The implications $(1) \Rightarrow(2) \Rightarrow(3) \Rightarrow(4)$ (and $(4) \Rightarrow(1)$ ) are a consequence of Theorem 2.3 .

(4) $\Rightarrow(5)$. This follows from the fact that $\mathbb{N} \subset \mathbb{Z}$ is equidecomposable with a proper subset of itself and [3, Lemma 3.2].

$(5) \Rightarrow(1)$. This is a consequence of [8, Lemma 1].

Remark 2.6. After this note was made available on arXiv, we became aware of [7, where it is shown that if a group is infinite and finitely generated, then its uniform Roe algebra is infinite.

Any locally finite group acts on itself in a transitive, faithful and locally finite way. If a finitely generated group admits a faithful, transitive, locally finite action on a set, then the group is finite. This is in stark contrast to the fact that there are finitely generated, non-amenable groups which admit faithful, transitive, amenable actions on sets (see [1] for various examples).

A finitely generated group admits a faithful, locally finite action if and only if it is residually finite.

Proposition 2.7. If a group admits a faithful, locally finite action, then it embeds into a group which admits a faithful, locally finite and transitive action.

Proof. Let $G$ be a group which acts on a set $X$ in a faithful and locally finite way.

Take a set $Y \subset X$ of representatives of all $G$-orbits, and let $S_{Y}$ be the group of finitely supported permutations of $Y$. Consider the natural action of $S_{Y}$ on $X$ and the associated action of $H:=G * S_{Y}$ on $X$. This action is transitive and locally finite. By taking the quotient of $H$ by the kernel of this action, we get a faithful, transitive, locally finite action on $X$ by a group which contains $G$. 
In analogy to what is known for amenable actions ([2, Lemma 3.2]), the following holds for locally finite actions:

Proposition 2.8. Let $G$ be a group acting on a set $X$ in a locally finite way. If, for every $x \in X$, the stabilizer subgroup $G_{x}$ is locally finite, then $G$ is locally finite.

Proof. Take $H<G$ finitely generated and $x \in X$. Since the action is locally finite, it follows that $H x$ is finite. Hence, there is $H_{0}$ a subgroup of finite index in $H$ such that $H_{0}<G_{x}$. In particular, $H_{0}$ is locally finite. Therefore, $H$ is also locally finite. Since $H$ is finitely generated, we conclude that it is finite.

Remark 2.9. One can define in a natural way an action of a group on a set $X$ to be supramenable if no subset of $X$ is equidecomposable with two disjoint proper subsets of itself. It is not true that if the action of a group $G$ is supramenable, and all the stabilizer subgroups are supramenable, then $G$ is supramenable.

Indeed, it is well-known that the class of supramenable groups is not closed by taking extensions (the lamplighter group $\mathbb{Z}_{2} \prec \mathbb{Z}$ is such an example). Let then $G$ be a non-supramenable group which contains a supramenable normal subgroup $N$ such that $\frac{G}{N}$ is also supramenable.

Consider the left action of $G$ on $\frac{G}{N}$. Since $\frac{G}{N}$ is supramenable, it follows easily that this action is supramenable. The stabilizer subgroups of the action are all equal to $N$, hence are supramenable.

Acknowledgement. The author thanks Claire Anantharaman-Delaroche for calling his attention to the reference [1].

\section{REFERENCES}

[1] Glasner, Y., and Monod, N. Amenable actions, free products and a fixed point property. Bulletin of the London Mathematical Society 39, 1 (2007), 138-150.

[2] Juschenko, K., And Monod, N. Cantor systems, piecewise translations and simple amenable groups. Annals of Mathematics 178, 2 (2013), 775-787.

[3] Kellerhals, J., Monod, N., and Rørdam, M. Non-supramenable groups acting on locally compact spaces. Documenta Mathematica 18 (2013), 1597-1626.

[4] Shalom, Y. Harmonic analysis, cohomology, and the large-scale geometry of amenable groups. Acta Mathematica 192, 2 (2004), 119-185.

[5] Silva, P. V., And Soares, F. Howson's property for semidirect products of semilattices by groups. Communications in Algebra 44, 6 (2016), 2482-2494.

[6] Wagon, S. The Banach-Tarski Paradox, vol. 24. Cambridge University Press, 1993.

[7] WeI, S. On the quasidiagonality of Roe algebras. Science China Mathematics 54, 5 (2011), 1011-1018.

[8] ŻUK, A. On an isoperimetric inequality for infinite finitely generated groups. Topology 39, 5 (2000), 947-956.

Department of Mathematical Sciences, University of Copenhagen,

Universitetsparken 5, DK-2100, Copenhagen, Denmark

E-mail address: duduscarparo@gmail.com 